\title{
Network Lifetime Maximization With Node Admission in Wireless Multimedia Sensor Networks
}

\author{
Khoa T. Phan, Student Member, IEEE, Rongfei Fan, Hai Jiang, Member, IEEE, \\ Sergiy A. Vorobyov, Senior Member, IEEE, and Chintha Tellambura, Senior Member, IEEE
}

\begin{abstract}
Wireless multimedia sensor networks (WMSNs) are expected to support multimedia services such as delivery of video and audio streams. However, due to the relatively stringent quality-of-service $(\mathrm{QoS})$ requirements of multimedia services (e.g., high transmission rates and timely delivery) and the limited wireless resources, it is possible that not all the potential sensor nodes can be admitted into the network. Thus, node admission is essential for WMSNs, which is the target of this paper. Specifically, we aim at the node admission and its interaction with power allocation and link scheduling. A cross-layer design is presented as a two-stage optimization problem, where at the first stage the number of admitted sensor nodes is maximized, and at the second stage the network lifetime is maximized. Interestingly, it is proved that the two-stage optimization problem can be converted to a one-stage optimization problem with a more compact and concise mathematical form. Numerical results demonstrate the effectiveness of the two-stage and one-stage optimization frameworks.
\end{abstract}

Index Terms-Admission control, cross-layer design, wireless multimedia sensor networks (WMSNs).

\section{INTRODUCTION}

$\mathbf{I}$ N ADDITION to low-rate delay-tolerant applications, wireless sensor networks (WSNs) are also expected to support multimedia services such as delivery of video and audio streams, which are referred to as wireless multimedia sensor networks (WMSNs) [1]. The WMSNs are expected to support quality-of-service (QoS) such as relatively high data rate and timely delivery. On one hand, similar to the case in traditional WSNs, resource management protocols such as power allocation, link scheduling, and routing in WMSNs interact with each other and, thus, should be jointly designed in a cross-layer manner. On the other hand, the stringent QoS requirements of multimedia services determine that node admission is necessary in WMSNs. Indeed, in traditional WSNs, the sensor nodes can be densely deployed with no need to predetermine their positions, and thus, node admission is unnecessary [2]. However, this may not work for WMSNs, since the limited bandwidth may not be able to support the high rate and timely multimedia

Manuscript received July 27, 2008; revised December 25, 2008. First published January 19, 2009; current version published August 14, 2009. This work was supported in part by the Alberta Ingenuity Fund, Alberta, Canada, and in part by the Natural Science and Engineering Research Council of Canada. The review of this paper was coordinated by Dr. E. Hossain.

K. T. Phan is with the Department of Electrical Engineering, California Institute of Technology, Pasadena, CA 91125 USA (e-mail: kphan@ caltech.edu).

R. Fan, H. Jiang, S. A. Vorobyov, and C. Tellambura are with the Department of Electrical and Computer Engineering, University of Alberta, Edmonton, AB T6G 2V4, Canada (e-mail: rongfei@ualberta.ca; hai.jiang@ece.ualberta.ca; vorobyov@ece.ualberta.ca; chintha@ece.ualberta.ca).

Digital Object Identifier 10.1109/TVT.2009.2013235 services from all potential sensor nodes. Thus, the problem of admitting as many potential sensor nodes as possible arises in addition to the aforementioned resource management problems (power allocation/link scheduling/routing). Furthermore, node admission and resource management also interact with each other. On one hand, node admission largely depends on the system capacity in the network, which is further affected by resource management. On the other hand, node admission determines the traffic load of the network, which apparently affects the resource management as well. In this paper, the node admission in WMSNs and its interaction with resource management protocols in a cross-layer design model are investigated.

In the existing literature, the cross-layer design of resource management protocols including power allocation, link scheduling, and routing has attracted tremendous attention [3], [4]. A joint routing/link scheduling/power control algorithm based on the minimization of the total average power consumption is presented in [5] for multihop wireless networks. An algorithm is proposed to find subsets of concurrently active links. In [6], a joint link-scheduling and power-control scheme is presented to achieve maximal network throughput, and a polynomial-time heuristic algorithm is developed to solve the problem when fairness is also considered. In [7], the joint design for finding the transmission power and rate in the physical layer, link schedule in the medium access control (MAC) layer, and routing in the network layer is presented to maximize the lifetime of a WSN, with the assumption that each sensor node has the flexibility to adapt its transmission power, modulation scheme, and duty cycle. Similarly, a joint design of routing and MAC is given in [8] to achieve the maximal network lifetime in WSNs. A distributed primal-dual algorithm is provided, in which the primal and dual domains deal with the MAC and routing, respectively. The network lifetime maximization problem is addressed in [9] and [10] for sensor networks with dynamic environments and under different network lifetime criteria. In [11], a joint design of MAC, routing, and energy distribution is investigated for multihop wireless networks.

Different from the aforementioned research efforts, we aim at the node admission that is necessary in WMSNs and its interaction with the aforementioned joint design of power allocation and link scheduling. Specifically, the interaction is formulated as a two-stage optimization problem that admits as many potential sensor nodes as possible and, meanwhile, achieves the maximal network lifetime. Interestingly, we prove that the two-stage optimization problem is equivalent to a onestage optimization problem. Our approach is applicable for networks containing nodes with low mobility. An example of 
such networks is a WMSN for multimedia sharing with fixed user locations.

The rest of this paper is organized as follows. The network model is given in Section II. The two-stage and equivalent one-stage cross-layer designs with node admission in WMSNs are presented in Section III. Numerical results are given in Section IV, followed by concluding remarks in Section V.

\section{NETWORK MODEL}

For a WMSN, let $\mathcal{N}$ denote the set of potential sensor nodes. If admitted, each node not only transmits its own traffic but also helps relay traffic from its neighboring nodes to the traffic sink. Therefore, multihop relay is assumed. Let $\mathcal{L}$ denote the set of unidirectional one-hop links in the WMSN. Each potential sensor node $n \in \mathcal{N}$, if admitted, generates traffic at a source rate $r_{n}$.

All the admitted sensor nodes share a single frequency band. Hence, each node cannot simultaneously transmit and receive. Further, a unicast network is assumed, i.e., among all the outgoing links from a node, at most one can be active at a time. In this context, a time-division multiple-access (TDMA) schedule is feasible for the admitted nodes to transmit. In a TDMA schedule, time is partitioned into fixed-length frames, and a frame consists of $J$ time slots. The resource allocation schedule repeats from one frame to another. In a frame, an admitted node can be active in one or more slots. When active at a slot, a node can transmit at a unit rate with the transmission power ranging from 0 to $P_{\max }$. Specifically, let $s_{j}^{l}=1$ indicate that link $l \in \mathcal{L}$ is active at slot $j \in \mathcal{J}=\{1,2, \ldots, J\}$, and let the corresponding transmission power be $P_{j}^{l} \in\left(0, P_{\max }\right]$. Similarly, let $s_{j}^{l}=0$ indicate that link $l$ is inactive at slot $j$, and thus, the corresponding transmission power is $P_{j}^{l}=0$. For a node $n$, let $\mathcal{O}(n)$ and $\mathcal{I}(n)$ denote the sets of outgoing and incoming links, respectively. The difference between an admitted node's outgoing and incoming traffic is exactly the source traffic from that node, i.e.,

$$
\sum_{l \in \mathcal{O}(n)} \sum_{j=1}^{J} s_{j}^{l} / J-\sum_{l \in \mathcal{I}(n)} \sum_{j=1}^{J} s_{j}^{l} / J=r_{n}, \quad n \in \mathcal{N} .
$$

Let $E_{n}$ denote the initial energy supply at node $n$. Thus, if node $n$ is admitted, then we have

$$
T_{n} \sum_{l \in \mathcal{O}(n)} \sum_{j=1}^{J} P_{j}^{l} t_{\text {slot }} \leq E_{n}
$$

where $t_{\text {slot }}$ is the duration of a time slot, and $T_{n}$ is the lifetime (unit: time frames) of node $n$. Here, we do not consider the energy consumption associated with packet reception, which can actually be straightforwardly added to the problem formulation.

The network lifetime $T_{\text {net }}$ (unit: time frames) is defined as the maximal time duration when all the admitted nodes function well, i.e., the time until the moment when the first sensor node dies. This definition is particularly feasible for WMSNs, since in many WMSN applications such as traffic avoidance/control systems and health care delivery systems [1], the data from each sensor node are required at the traffic sink. Thus, it is critical to keep all the admitted sensor nodes functioning well during the lifetime of the network.

In the WMSN, each active link should be guaranteed with a certain level of transmission accuracy. Thus, a minimum signal-to-interference-plus-noise ratio (SINR) is required for each active link. Specifically, for $s_{j}^{l}=1$, we should have

$$
\operatorname{SINR}_{j}^{l}=\frac{P_{j}^{l} h_{l l}}{\sum_{k \in \mathcal{L}, k \neq l} P_{j}^{k} h_{k l}+\eta_{l}} \geq \Gamma
$$

where $\operatorname{SINR}_{j}^{l}$ is the $\operatorname{SINR}$ for link $l$ at slot $j, h_{k l}$ is the path gain from link $k$ 's transmitter to link $l$ 's receiver, $\eta_{l}$ is the background noise power at link $l$ 's receiver, and $\Gamma$ is the required SINR threshold.

\section{Network LifEtime MaXimization WiTH NODE ADMISSION}

\section{A. Cross-Layer Optimization Framework Without Node Admission}

Cross-layer optimization is a powerful tool for the design of power allocation and link scheduling in multihop wireless networks [12], [13]. For a WMSN, if all the potential sensor nodes are admitted, then a network lifetime maximization problem can be formulated as follows:

$$
\max _{\left\{P_{j}^{l}\right\},\left\{s_{j}^{l}\right\}, T_{\text {net }}} T_{\text {net }}
$$

subject to:

$$
\begin{aligned}
& T_{\text {net }} \sum_{l \in \mathcal{O}(n)} \sum_{j=1}^{J} P_{j}^{l} t_{\text {slot }} \leq E_{n}, \quad n \in \mathcal{N} \\
& \sum_{l \in \mathcal{O}(n)} \sum_{j=1}^{J} s_{j}^{l}-\sum_{l \in \mathcal{I}(n)} \sum_{j=1}^{J} s_{j}^{l}=J r_{n}, \quad n \in \mathcal{N} \\
& \sum_{l \in \mathcal{O}(n)} s_{j}^{l}+\sum_{l \in \mathcal{I}(n)} s_{j}^{l} \leq 1, \quad n \in \mathcal{N}, \quad j \in \mathcal{J} \\
& P_{j}^{l} h_{l l} \geq \Gamma \cdot \sum_{k \in \mathcal{L}, k \neq l} P_{j}^{k} h_{k l}+\Gamma \eta_{l} \\
& \quad+C\left(s_{j}^{l}-1\right), \quad l \in \mathcal{L}, \quad j \in \mathcal{J} \\
& 0 \leq P_{j}^{l} \leq s_{j}^{l} P_{\max }, \quad l \in \mathcal{L}, \quad j \in \mathcal{J} \\
& s_{j}^{l} \in\{0,1\}, \quad l \in \mathcal{L}, \quad j \in \mathcal{J}
\end{aligned}
$$

where the constraint (3d) means that, at any time, at most one link with node $n$ is active, and the constraint (3e) is equivalent to (2), in which $C$ is a constant that satisfies

$$
C \geq \Gamma(|\mathcal{L}|-1) P_{\max } \cdot \max _{k, l}\left\{h_{k l}\right\}+\Gamma \cdot \max _{l}\left\{\eta_{l}\right\}
$$

where $|\mathcal{L}|$ denotes the number of elements in $\mathcal{L}$. Constraint (3e) is equivalent to constraint (2) for the following reason. For 
constraint (3e), we have the following.

1) When link $l$ is scheduled to be activate in time slot $j$, i.e., $s_{j}^{l}=1$, constraint ( $3 \mathrm{e}$ ) can exactly be rewritten as constraint (2).

2) When link $l$ is not scheduled in time slot $j$, i.e., $s_{j}^{l}=0$, the constraint $(3 e)$ is satisfied at any scenario as long as the constant $C$ is larger than the lower bound given earlier.

Therefore, constraints (2) and (3e) are equivalent.

Introducing a new variable

$$
B=1 / T_{\text {net }}
$$

the optimization problem (3a)-(3g) can be rewritten as

$$
\min _{\left\{P_{j}^{l}\right\},\left\{s_{j}^{l}\right\}, B} B
$$

subject to:

$$
\sum_{l \in \mathcal{O}(n)} \sum_{j=1}^{J} P_{j}^{l} \leq \frac{E_{n}}{t_{\text {slot }}} \cdot B, \quad n \in \mathcal{N}
$$

Constraints $(3 \mathrm{c})-(3 \mathrm{~g})$

which is a mixed-integer linear programming (MILP) problem.

\section{B. Network Lifetime Maximization With Node Admission}

1) Two-Stage Optimization: The optimization problem (4a)-(4c) may not have a feasible solution. In other words, there may not exist $\left(\left\{P_{j}^{l}\right\},\left\{s_{j}^{l}\right\}, B\right)$ that satisfies the constraints (4b) and (4c), e.g., the source rates $r_{n}$ 's of the nodes cannot be achieved at any TDMA schedule. This may be due to the limited communication resources, for example, when the spectrum bandwidth is not sufficient. Therefore, node admission is necessary when the admitted nodes should be guaranteed with their requested source rates. To ensure the coverage of the WMSN, it is required that some selected nodes should always be admitted and guaranteed with their source rates, which are referred to as higher-priority nodes. ${ }^{1}$ The set of higher-priority nodes is denoted as $\tilde{\mathcal{N}}$. The other nodes are referred to as lowerpriority nodes. It is assumed that the source rate requirements of higher-priority nodes can always be guaranteed through the available network resources. Then, the objective of node admission is to find the maximal number $N^{+}$of lower-priority nodes that can be admitted into the WMSN. For each node $n \in \mathcal{N} \backslash \tilde{\mathcal{N}}$, define $y_{n}$ as the activity factor where $y_{n}=1$ if node $n$ is admitted and $y_{n}=0$ otherwise. After the maximal number $\mathrm{N}^{+}$is determined, it is also necessary to maximize the network lifetime $T_{\text {net }}$. Therefore, the aforementioned strategy can be expressed in mathematical form as the following twostage optimization problem.

\footnotetext{
${ }^{1}$ Alternatively, it can also be required that at least one node should be admitted from each small group of nodes. This case can be shown to be equivalent to the case with higher priority nodes.
}

Stage 1-Node admission control:

$$
\max _{\left\{P_{j}^{l}\right\},\left\{s_{j}^{l}\right\},\left\{y_{n}\right\}} \sum_{n \in \mathcal{N} \backslash \tilde{\mathcal{N}}} y_{n}
$$

subject to

$$
\begin{aligned}
& \sum_{l \in \mathcal{O}(n)} \sum_{j=1}^{J} s_{j}^{l}-\sum_{l \in \mathcal{I}(n)} \sum_{j=1}^{J} s_{j}^{l}=J r_{n}, \quad n \in \tilde{\mathcal{N}} \\
& \sum_{l \in \mathcal{O}(n)} \sum_{j=1}^{J} s_{j}^{l}-\sum_{l \in \mathcal{I}(n)} \sum_{j=1}^{J} s_{j}^{l}=y_{n} J r_{n}, \quad n \in \mathcal{N} \backslash \tilde{\mathcal{N}} \\
& \sum_{l \in \mathcal{I}(n)} \sum_{j=1}^{J} s_{j}^{l} \leq y_{n} D, \quad n \in \mathcal{N} \backslash \tilde{\mathcal{N}} \\
& y_{n} \in\{0,1\}, \quad n \in \mathcal{N} \backslash \tilde{\mathcal{N}} \\
& \text { Constraints (3d)-(3g). }
\end{aligned}
$$

The constraint (5b) means that all the higher-priority nodes should be admitted. If a lower-priority node $n$ is not admitted, then $y_{n}=0$, the constraint $(5 \mathrm{c})$ means that node $n$ does not inject traffic into the network, and the constraint $(5 \mathrm{~d})$ means that node $n$ does not relay traffic for its neighboring nodes, where $D$ is a constant that satisfies

$$
D \geq J \cdot \max _{n \in \mathcal{N} \backslash \tilde{\mathcal{N}}}|\mathcal{I}(n)|
$$

In other words, lower-priority nodes, if not admitted, will not participate in the multihop relay services. The problem (5a)-(5f) is also an MILP problem. Note that the solution to the optimization problem (5a)-(5f) (i.e., the optimal set of admitted lower-priority nodes) that provides maximal number of admitted lower-priority nodes (i.e., $N^{+}$) is not unique in general. Hence, among the multiple solutions, i.e., the multiple admitted lower-priority node sets (each of which has totally $N^{+}$ lower-priority nodes), it is desired to select a set that leads to the maximal network lifetime, as shown in the second stage.

Stage 2-Network lifetime maximization: For each set of admitted lower-priority nodes obtained from the first stage, the following subproblem needs to be solved:

$$
\min _{\left\{P_{j}^{l}\right\},\left\{s_{j}^{l}\right\}, B} B
$$

subject to:

$$
\text { Constraints (4b), (5b)-(5d), and (5f). }
$$

Among all the admitted lower-priority node sets, we select the set whose associated subproblem (6a)-(6b) has the smallest optimal $B$ value. The power allocation and transmission schedule of the admitted node set (i.e., $\left\{P_{j}^{l}, s_{j}^{l}\right\}$ ) can also be obtained by solving the associated subproblem.

It can be seen from the design process that the design priority is to admit as many nodes as possible. We select the set of nodes that has the largest number of elements and, at the same time, leads to the maximal network lifetime. A similar 
node-admission principle has first been used in [14] for downlink cellular beamforming.

2) Equivalent One-Stage Optimization: The following theorem is in order.

Theorem 1: The two-stage optimization problem (5a)-(5f) and $(6 a)-(6 b)$ is equivalent to the following one-stage optimization problem:

$$
\max _{\left\{P_{j}^{l}\right\},\left\{s_{j}^{l}\right\},\left\{y_{n}\right\}, B} \beta \sum_{n \in \mathcal{N} \backslash \tilde{\mathcal{N}}} y_{n}-(1-\beta) B
$$

subject to

$$
\text { Constraints (4b) and (5b)-(5f) }
$$

where

$$
\beta \in\left[\frac{1}{1+T_{\min }}, 1\right)
$$

is a constant

$$
T_{\min }=\min _{n \in \mathcal{N}} \frac{E_{n}}{|\mathcal{O}(n)| J P_{\max } t_{\text {slot }}}
$$

and $|\mathcal{O}(n)|$ denotes the number of elements in $\mathcal{O}(n)$.

Proof: The proof consists of three steps.

In the first step, we prove that the solution of the one-stage problem (7a)-(7b) and the solution of the problem (5a)-(5f) in Stage 1 of the two-stage problem will give the same maximum number of admitted lower-priority nodes.

Let $\left(\mathcal{N}^{+}, \mathcal{P}^{+}, \mathcal{S}^{+}\right)$be an optimal solution to the problem (5a)-(5f), with $\mathcal{N}^{+}$being the set of admitted lower-priority nodes, $\mathcal{P}^{+}=\left\{P_{j}^{l^{+}}, \quad \forall l \in \mathcal{L}, j \in \mathcal{J}\right\}$ and $\mathcal{S}^{+}=\left\{s_{j}^{l^{+}}, \quad \forall l \in\right.$ $\mathcal{L}, j \in \mathcal{J}\}$ being the corresponding power allocation and transmission schedule, respectively, and the optimal objective value being $\left|\mathcal{N}^{+}\right|=N^{+} .^{2}$ Similarly, let $\left(\mathcal{N}^{*}, \mathcal{P}^{*}, \mathcal{S}^{*}, B^{*}\right)$ be an optimal solution to the one-stage optimization problem (7a)-(7b), with $\mathcal{N}^{*}$ being the set of admitted lower-priority nodes, $\mathcal{P}^{*}$ and $\mathcal{S}^{*}$ being the corresponding power allocation and transmission schedule, respectively, $B^{*}$ being the inverse network lifetime, and the number of admitted lower-priority nodes being $\left|\mathcal{N}^{*}\right|=$ $N^{*}$. The optimal objective value of the problem (7a)-(7b) is given by

$$
\mathcal{V}^{*}=\beta N^{*}-(1-\beta) B^{*}
$$

In the following, we prove using contradiction that $N^{*}=N^{+}$.

Suppose $N^{*}<N^{+}$. Since both $N^{*}$ and $N^{+}$are integers, we have

$$
N^{+}-N^{*} \geq 1
$$

Define

$$
B^{+}=\max _{n \in \mathcal{N}} \frac{\sum_{l \in \mathcal{O}(n)} \sum_{j=1}^{J} P_{j}^{l^{+}} t_{\text {slot }}}{E_{n}} .
$$

\footnotetext{
${ }^{2}$ Note that there may be many optimal solutions for problem (5a)-(5f) with the same optimal value $N^{+}$. Therefore, $\left(\mathcal{N}^{+}, \mathcal{P}^{+}, \mathcal{S}^{+}\right)$here is just one of them.
}

Then, we have

$$
B^{+} \leq \max _{n \in \mathcal{N}} \frac{|\mathcal{O}(n)| J P_{\max } t_{\text {slot }}}{E_{n}}=\frac{1}{T_{\min }} .
$$

Apparently, $\left(\mathcal{N}^{+}, \mathcal{P}^{+}, \mathcal{S}^{+}, B^{+}\right)$satisfies constraints (4b) and (5b)-(5f), and thus, it is a feasible solution to the one-stage optimization problem (7a)-(7b), with the objective value being

$$
\mathcal{V}^{+}=\beta N^{+}-(1-\beta) B^{+} .
$$

Further, we have

$$
\begin{aligned}
\mathcal{V}^{+}-\mathcal{V}^{*} & =\beta\left(N^{+}-N^{*}\right)+(1-\beta)\left(B^{*}-B^{+}\right) \\
& >\beta-(1-\beta) \frac{1}{T_{\min }} \\
& =\left(1+\frac{1}{T_{\min }}\right) \beta-\frac{1}{T_{\min }} \\
& \geq\left(1+\frac{1}{T_{\min }}\right) \frac{1}{1+T_{\min }}-\frac{1}{T_{\min }} \\
& =0 .
\end{aligned}
$$

The first inequality in (11) holds because

$$
\begin{aligned}
& N^{+}-N^{*} \geq 1 \\
& B^{*}-B^{+}>-B^{+} \geq-1 / T_{\min }
\end{aligned}
$$

and the second inequality in (11) holds because

$$
\beta \in\left[\frac{1}{1+T_{\min }}, 1\right) .
$$

Inequality (11) contradicts the fact that $\left(\mathcal{N}^{*}, \mathcal{P}^{*}, \mathcal{S}^{*}, B^{*}\right)$ is an optimal solution to the one-stage problem (7a)-(7b). Therefore, we should have $N^{*} \geq N^{+}$.

On the other hand, since $\left(\mathcal{N}^{*}, \mathcal{P}^{*}, \mathcal{S}^{*}, B^{*}\right)$ is an optimal solution to the problem $(7 \mathrm{a})-(7 \mathrm{~b}),\left(\mathcal{N}^{*}, \mathcal{P}^{*}, \mathcal{S}^{*}\right)$ satisfies the constraints $(5 \mathrm{~b})-(5 \mathrm{f})$. This means that $\left(\mathcal{N}^{*}, \mathcal{P}^{*}, \mathcal{S}^{*}\right)$ is also a feasible solution to the problem (5a)-(5f). Since $\left(\mathcal{N}^{+}, \mathcal{P}^{+}, \mathcal{S}^{+}\right)$ is an optimal solution to the problem (5a)-(5f), we have $\left|\mathcal{N}^{+}\right|=N^{+} \geq\left|\mathcal{N}^{*}\right|=N^{*}$. Together with the fact that $N^{*} \geq$ $N^{+}$, it can be concluded that $N^{*}=N^{+}$.

In the second step, we prove that the optimal power allocation, transmission schedule, and inverse network lifetime $\left(\mathcal{P}^{*}, \mathcal{S}^{*}, B^{*}\right)$ obtained from the one-stage problem (7a)-(7b) is also an optimal solution to the subproblem (6a)-(6b) associated with the admitted lower-priority node set $\mathcal{N}^{*}$. We again use proof by contradiction. Suppose $\left(\mathcal{P}^{\dagger}, \mathcal{S}^{\dagger}, B^{\dagger}\right)$ is an optimal solution to the subproblem (6a)-(6b) associated with the admitted lower-priority node set $\mathcal{N}^{*}$, and $B^{\dagger}<B^{*}$. Since $\left(\mathcal{N}^{*}, \mathcal{P}^{\dagger}, \mathcal{S}^{\dagger}, B^{\dagger}\right)$ satisfies (6b), it also satisfies (7b). Thus, it is a feasible solution to the one-stage problem (7a)-(7b), with the objective value being

$$
\mathcal{V}^{\dagger}=\beta N^{*}-(1-\beta) B^{\dagger}>\beta N^{*}-(1-\beta) B^{*}=\mathcal{V}^{*}
$$

which contradicts the fact that $\left(\mathcal{N}^{*}, \mathcal{P}^{*}, \mathcal{S}^{*}, B^{*}\right)$ is an optimal solution to the problem $(7 \mathrm{a})-(7 \mathrm{~b})$. Therefore, it can be 
concluded that $\left(\mathcal{P}^{*}, \mathcal{S}^{*}, B^{*}\right)$ is an optimal solution to the subproblem (6a)-(6b) associated with the admitted lower-priority node set $\mathcal{N}^{*}$.

In the third step, we prove that $B^{*}$ is the smallest value among the (multiple) optimal values of the (multiple) subproblems (6a)-(6b) when the number of admitted lower-priority nodes is $N^{*}\left(=N^{+}\right)$. We still use proof by contradiction. Suppose $B^{*}$ is not the smallest value. Therefore, there exists an admitted lower-priority node set $\mathcal{N}^{\ddagger}$ such that $\left|\mathcal{N}^{\ddagger}\right|=N^{*}$ and $B^{\ddagger}<B^{*}$, where $\left(\mathcal{P}^{\ddagger}, \mathcal{S}^{\ddagger}, B^{\ddagger}\right)$ is an optimal solution to the subproblem (6a)-(6b) associated with $\mathcal{N}^{\ddagger}$. Apparently, $\left(\mathcal{N}^{\ddagger}, \mathcal{P}^{\ddagger}, \mathcal{S}^{\ddagger}, B^{\ddagger}\right)$ satisfies (7b), and thus, it is a feasible solution to the problem (7a)-(7b), with the objective value being

$$
\mathcal{V}^{\ddagger}=\beta N^{*}-(1-\beta) B^{\ddagger}>\beta N^{*}-(1-\beta) B^{*}=\mathcal{V}^{*}
$$

which contradicts the fact that $\left(\mathcal{N}^{*}, \mathcal{P}^{*}, \mathcal{S}^{*}, B^{*}\right)$ is an optimal solution to the problem (7a)-(7b). Thus, $B^{*}$ must be the smallest value among the (multiple) optimal values of the (multiple) subproblems (6a)-(6b).

From the preceding three steps, it can be concluded that the two-stage optimization problem (5a)-(5f) and (6a)-(6b) is equivalent to the one-stage optimization problem (7a)-(7b). This completes the proof.

Lemma 1: The one-stage optimization problem (7a)-(7b) is always feasible.

Proof: In the worst case, only higher-priority nodes are admitted. This means that $y_{n}=0, \forall n \in \mathcal{N} \backslash \tilde{\mathcal{N}}$. Then, the one-stage optimization problem is to maximize the network lifetime when only higher-priority nodes are present. This problem is always feasible since it is assumed that the source rate requirements of higher-priority nodes can always be guaranteed through available network resources.

Both one-stage and two-stage optimization problems are MILP problems and, thus, are NP-hard and have worst-case exponential complexity. The complexity of either problem depends on the dimension that further depends on the network size and topology, the size of the time slots, the number of links, and the data rate requirements. A more detailed complexity analysis is likely intractable. However, only one NP-hard problem is involved in the one-stage implementation, whereas a number of such problems are involved in the two-stage implementation. Solving only one problem reduces the worst-case computation time. In the literature, some standard iterative methods have been developed to solve this class of problems, such as the cutting-plane method, the branch-and-bound method, the branch-and-cut method, etc. [15]. Software packages such as CPLEX [16] are also available to deal with the proposed MILP problems.

Either the two-stage or the one-stage problem with node admission should be solved in a centralized manner. Therefore, the proposed design applies to small- or medium-size WMSNs. For a large-size WMSN, a possible solution is to divide the network into clusters (by methods discussed in [17] and references therein) and apply the proposed design in each cluster. Therefore, the target network of the proposed design is a smallor medium-size WMSN or a cluster in a large-size WMSN.

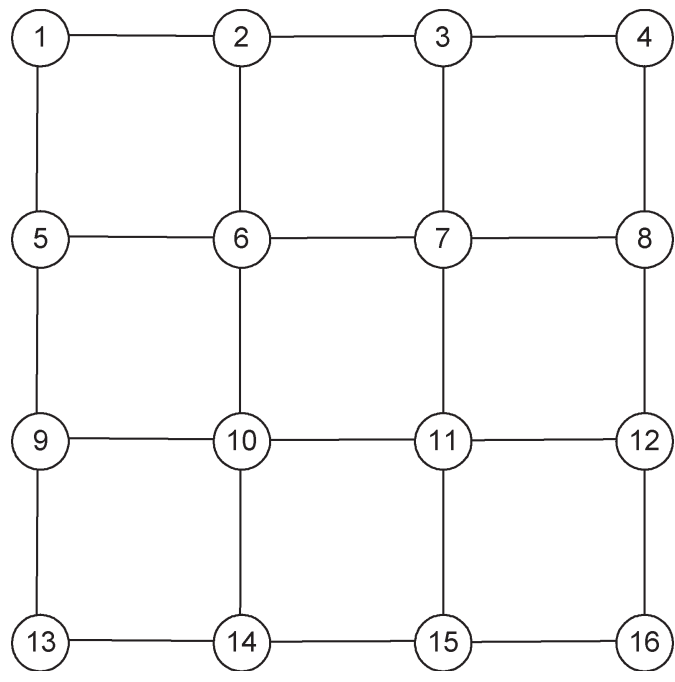

Fig. 1. Network topology.

\section{Numerical Results}

Consider a WMSN as shown in Fig. 1. The WMSN has 16 nodes, among which nodes 7, 10, and 11 are the higherpriority nodes. The distance between two neighboring nodes is $100 \mathrm{~m}$. When active at a slot, a node transmits at a unit rate being $2.4 \mathrm{Mb} / \mathrm{s}$. The maximal transmission power of each node is $P_{\max }=0.1 \mathrm{~W}$. For the wireless signal propagation model, we assume that there is no shadowing or fading, and the power at the receiver is attenuated due to path loss, with the path attenuation exponent being equal to 4 . In the TDMA schedule, each time frame consists of 24 time slots, i.e., $J=24$. The duration of each time slot is $t_{\text {slot }}=5 \mathrm{~ms}$. Each higherpriority node requests a source rate of $200 \mathrm{~kb} / \mathrm{s}$. The required SINR threshold for each admitted node is $\Gamma=6$. Each node is equipped with a battery with power of $5000 \mathrm{~J}$. The background noise power is $-90 \mathrm{dBm}$. The software package CPLEX is used to find the solutions of the two-stage and one-stage problems.

It is observed that the two-stage and one-stage problems have the same optimal solution. Tables I-III show the number of admitted lower-priority nodes $N^{*}$, the admitted lower-priority node set $\mathcal{N}^{*}$, the maximal network lifetime $T_{\text {net }}^{*}$, and the computation time $T_{\text {com }}$ (i.e., the time needed by a computer with a 2.4-GHz CPU to solve the one-stage optimization problem) for the cases with the traffic sink being nodes 1,2 , and 6 , respectively. It can be seen that, in each case, when the source rate of a lower-priority node increases, the number of admitted lowerpriority nodes decreases. However, the network lifetime does not decrease in general, since the number of admitted lowerpriority nodes may decrease. When the traffic sink changes from node 1 to node 6 , we can see that the network performance (in terms of the number of admitted lower-priority nodes and/or network lifetime) is improved. This is because node 1 is at the corner of the network and, thus, is likely to become a bottleneck. It can also be seen that the computation time may be affected by the network topology, the location of the traffic sink, and the rate requirements of the nodes. Therefore, it is hard to indicate an upper bound of the network size for which CPLEX can work well. However, it is reasonable to expect that CPLEX 
TABLE I

Number of Admitted Lower-Priority Nodes $\left(N^{*}\right)$, Admitted Lower-Priority Node Set $\left(\mathcal{N}^{*}\right)$, MaXimal Network Lifetime $\left(T_{\text {net }}^{*}\right)$, and Computation Time $\left(T_{\text {com }}\right)$ in the Case With the Traffic Sink Being Node 1

\begin{tabular}{|c|c|c|c|c|}
\hline $\begin{array}{c}\text { Rate of a } \\
\text { lower-priority node }\end{array}$ & $N^{*}$ & $\mathcal{N}^{*}$ & $\begin{array}{c}T_{\text {net }}^{*} \\
\text { (seconds) }\end{array}$ & $\begin{array}{c}T_{\text {com }} \\
\text { (seconds) }\end{array}$ \\
\hline $100 \mathrm{kbps}$ & 12 & $\{2,3,4,5,6,8,9,12,13,14,15,16\}$ & $2.27 \times 10^{6}$ & 196 \\
\hline $200 \mathrm{kbps}$ & 5 & $\{2,3,4,5,9\}$ & $6.38 \times 10^{6}$ & 166 \\
\hline $400 \mathrm{kbps}$ & 4 & $\{2,3,5,9\}$ & $2.71 \times 10^{6}$ & 33 \\
\hline $500 \mathrm{kbps}$ & Not feasible & - & - & -
\end{tabular}

TABLE II

Number of Admitted Lower-Priority Nodes $\left(N^{*}\right)$, Admitted Lower-Priority Node Set $\left(\mathcal{N}^{*}\right)$, MaXimal Network Lifetime $\left(T_{\text {net }}^{*}\right)$, and Computation Time $\left(T_{\text {com }}\right)$ in the Case With the Traffic Sink Being Node 2

\begin{tabular}{|c|c|c|c|c|}
\hline $\begin{array}{c}\text { Rate of a } \\
\text { lower-priority node }\end{array}$ & $N^{*}$ & $\mathcal{N}^{*}$ & $\begin{array}{c}T_{\text {net }}^{*} \\
\text { (seconds) }\end{array}$ & $\begin{array}{c}T_{\text {com }} \\
\text { (seconds) }\end{array}$ \\
\hline $100 \mathrm{kbps}$ & 12 & $\{1,3,4,5,6,8,9,12,13,14,15,16\}$ & $2.89 \times 10^{6}$ & 80 \\
\hline $200 \mathrm{kbps}$ & 7 & $\{1,3,4,5,6,8,14\}$ & $6.97 \times 10^{6}$ & 11918 \\
\hline $400 \mathrm{kbps}$ & 4 & $\{3,4,6,14\}$ & $4.36 \times 10^{6}$ & 258 \\
\hline $500 \mathrm{kbps}$ & 3 & $\{1,3,6\}$ & $1.10 \times 10^{7}$ & 180 \\
\hline
\end{tabular}

TABLE III

Number of Admitted Lower-Priority Nodes $\left(N^{*}\right)$, Admitted Lower-Priority Node Set $\left(\mathcal{N}^{*}\right)$, Maximal Network Lifetime $\left(T_{\text {net }}^{*}\right)$, and Computation Time $\left(T_{\text {com }}\right)$ In the CASE With the Traffic Sink Being Node 6

\begin{tabular}{|c|c|c|c|c}
\hline $\begin{array}{c}\text { Rate of a } \\
\text { lower-priority node }\end{array}$ & $N^{*}$ & $\mathcal{N}^{*}$ & $\begin{array}{c}T_{\text {net }}^{*} \\
\text { (seconds) }\end{array}$ & $\begin{array}{c}T_{\text {com }} \\
\text { (seconds) }\end{array}$ \\
\hline $100 \mathrm{kbps}$ & 12 & $\{1,2,3,4,5,8,9,12,13,14,15,16\}$ & $4.15 \times 10^{6}$ & 3 \\
\hline $200 \mathrm{kbps}$ & 9 & $\{1,2,3,4,5,8,9,14,15\}$ & $3.02 \times 10^{6}$ & 609 \\
\hline $400 \mathrm{kbps}$ & 4 & $\{2,5,8,14\}$ & $1.42 \times 10^{7}$ & 139 \\
\hline $500 \mathrm{kbps}$ & 3 & $\{2,5,14\}$ & $1.93 \times 10^{7}$ & 1918 \\
\hline
\end{tabular}

is likely to work well with the target network of the proposed design, i.e., a small- or medium-sized WMSN, or a cluster in a large-sized WMSN.

\section{CONCLUSION}

Unlike traditional WSNs, the WMSNs bring about new research challenges such as node admission. In this paper, node admission in WMSNs has been studied, and its interaction with power allocation and link scheduling has also been investigated. The two-stage optimization problem and the one-stage equivalent problem have been developed and demonstrated to be effective to obtain the desired performance in a WMSN. Overall, this paper has provided insights in where and how to deploy sensor nodes so that all the nodes can be supported by the limited communication resources in WMSNs.

\section{ACKNOWLEDGMENT}

The authors would like to thank the Associate Editor Prof. E. Hossain and the anonymous reviewers for their constructive comments and suggestions, which helped to improve the quality of the manuscript. This paper was completed when K. T. Phan was a graduate student at the Department of Electrical and Computer Engineering, University of Alberta, Edmonton, AB, Canada.

\section{REFERENCES}

[1] I. F. Akyildiz, T. Melodia, and K. R. Chowdury, "Wireless multimedia sensor networks: A survey," IEEE Wireless Commun., vol. 14, no. 6, pp. 32-39, Dec. 2007.

[2] I. F. Akyildiz, W. Su, Y. Sankarasubramaniam, and E. Cayirci, "Wireless sensor networks: A survey," Comput. Netw., vol. 38, no. 4, pp. 393-422, Mar. 2002.
[3] X. Shen, W. Zhuang, H. Jiang, and J. Cai, "Medium access control in ultra-wideband wireless networks," IEEE Trans. Veh. Technol., vol. 54, no. 5, pp. 1663-1677, Sep. 2005.

[4] H. T. Cheng, H. Jiang, and W. Zhuang, "Distributed medium access control for wireless mesh networks," Wirel. Commun. Mobile Comput., vol. 6, no. 6, pp. 845-864, Sep. 2006.

[5] R. L. Cruz and A. V. Santhanam, "Optimal routing, link scheduling and power control in multi-hop wireless networks," in Proc. IEEE INFOCOM, San Francisco, CA, Mar./Apr. 2003, pp. 702-711.

[6] J. Tang, G. Xue, C. Chandler, and W. Zhang, "Link scheduling with power control for throughput enhancement in multihop wireless networks," IEEE Trans. Veh. Technol., vol. 55, no. 3, pp. 733-742, May 2006.

[7] R. Madan, S. Cui, S. Lall, and A. Goldsmith, "Cross-layer design for lifetime maximization in interference-limited wireless sensor networks," IEEE Trans. Wireless Commun., vol. 5, no. 11, pp. 3142-3152, Nov. 2006.

[8] S.-J. Kim, X. Wang, and M. Madihian, "Distributed joint routing and medium access control for lifetime maximization of wireless sensor networks," IEEE Trans. Wireless Commun., vol. 6, no. 7, pp. 2669-2677, Jul. 2007.

[9] Y. Chen, Q. Zhao, V. Krishnamurthy, and D. Djonin, "Transmission scheduling for optimizing sensor network lifetime: A stochastic shortest path approach," IEEE Trans. Signal Process., vol. 55, no. 5, pp. 2294 2309, May 2007.

[10] Y. Chen and Q. Zhao, "On the lifetime of wireless sensor networks," IEEE Commun. Lett., vol. 9, no. 11, pp. 976-978, Nov. 2005.

[11] K. T. Phan, H. Jiang, C. Tellambura, S. A. Vorobyov, and R. Fan, "Joint medium access control, routing and energy distribution in multi-hop wireless networks," IEEE Trans. Wireless Commun., vol. 7, no. 12, pp. 5244 5249, Dec. 2008.

[12] X. Lin, N. B. Shroff, and R. Srikant, "A tutorial on cross-layer optimization in wireless networks," IEEE J. Sel. Areas Commun., vol. 24, no. 8 , pp. 1452-1463, Aug. 2006.

[13] L. Chen, S. H. Low, and J. C. Doyle, "Joint congestion control and media access control design for ad hoc wireless networks," in Proc. IEEE INFOCOM, Miami, FL, Mar. 2005, pp. 2212-2222.

[14] E. Matskani, N. D. Sidiropoulos, Z.-Q. Luo, and L. Tassiulas, "Convex approximation techniques for joint multiuser downlink beamforming and admission control," IEEE Trans. Wireless Commun., vol. 7, no. 7, pp. 2682-2693, Jul. 2008.

[15] A. Schrijver, Theory of Linear and Integer Programming. Hoboken, NJ: Wiley, 1998.

[16] [Online]. Available: http://tomopt.com/tomlab/

[17] O. Younis, M. Krunz, and S. Ramasubramanian, "Node clustering in wireless sensor networks: Recent developments and deployment challenges," IEEE Netw., vol. 20, no. 3, pp. 20-25, May/Jun. 2006. 


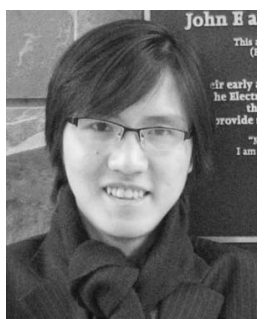

Khoa T. Phan (S'05) received the B.Sc. degree (with First-Class Honors) from the University of New South Wales, Sydney, NSW, Australia, in 2005 and the M.Sc. degree from the University of Alberta, Edmonton, AB, Canada, in 2008. He is currently working toward the Ph.D. degree with the Department of Electrical Engineering, California Institute of Technology, Pasadena.

His current research interests are mathematical foundations and control and optimization of communications networks. He is also interested in network economics, applications of game theory, and mechanism design in communications networks.

Mr. Phan has been awarded several prestigious fellowships, including the Australian Development Scholarship, the Alberta Ingenuity Fund Student Fellowship, the iCORE Graduate Student Award, and, most recently, the Atwood Fellowship, to name a few.

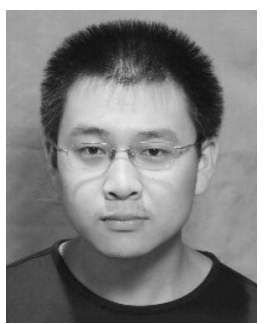

Rongfei Fan received the B.E. degree in electrical engineering from Harbin Institute of Technology, Harbin, China, in 2007. He is currently working toward the Ph.D. degree with the Department of Electrical and Computer Engineering, University of Alberta, Edmonton, AB, Canada.

His research interests include cognitive radio and cross-layer design.

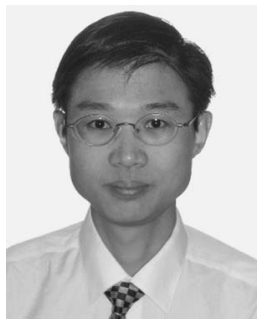

Hai Jiang (S'04-M'07) received the B.S. and M.S. degrees (with an Excellent Graduate Award) in electronics engineering from Peking University, Beijing, China, in 1995 and 1998, respectively, and the Ph.D. degree (with an Outstanding Achievement in Graduate Studies Award) in electrical engineering from the University of Waterloo, Waterloo, ON, Canada, in 2006.

From September 2006 to June 2007, he was a Postdoctoral Research Fellow with Princeton University, Princeton, NJ. Since July 2007, he has been an Assistant Professor with the Department of Electrical and Computer Engineering, University of Alberta, Edmonton, AB, Canada. His research interests include radio resource management, cellular/wireless local area network interworking, and cross-layer design for wireless multimedia communications.

Dr. Jiang is an Associate Editor for the IEEE TRANSACTIONS ON Vehicular TeChNOLOGY. He received an Alberta Ingenuity New Faculty Award in 2008 and a Best Paper Award from IEEE Global Communications Conference in 2008. He served or will serve as Cochair for the General Symposium at the 2007 International Wireless Communications and Mobile Computing Conference, the Communications and Networking Symposium at the 2009 Canadian Conference on Electrical and Computer Engineering, and the Wireless and Mobile Networking Symposium at the 2010 IEEE International Conference on Communications.

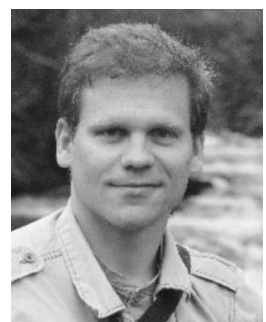

Sergiy A. Vorobyov (M'02-SM'05) received the M.S. and Ph.D. degrees from the National University of Radioelectronics, Kharkov, Ukraine, in 1994 and 1997, respectively.

$\mathrm{He}$ occupied research and faculty positions with the National University of Radioelectronics, Kharkiv, Ukraine; the Institute of Physical and Chemical Research (RIKEN), Japan; McMaster University, Hamilton, ON, Canada; Duisburg-Essen and Darmstadt Universities, Germany; and the Joint Research Institute, Heriot-Watt and Edinburgh Universities, Edinburgh, U.K. Since 2006, he has been an Assistant Professor with the Department of Electrical and Computer Engineering, University of Alberta, Edmonton, AB, Canada. His research interests include statistical array signal processing, applications of linear algebra and optimization methods in signal processing and communications, estimation and detection theory, sampling theory, multiantenna communications, and cooperative and cognitive systems.

Dr. Vorobyov currently serves as an Associate Editor for the IEEE Transactions on Signal Processing and the IEEE Signal PROCESSING LeTters. He is a member of Sensor Array and Multi-Channel Signal Processing Technical Committee of the IEEE Signal Processing Society. He was the recipient of the 2004 IEEE Signal Processing Society Best Paper Award, the 2007 Alberta Ingenuity New Faculty Award, and other research awards.

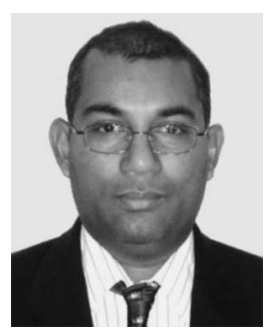

Chintha Tellambura (M'97-SM'02) received the B.Sc. degree (with first-class honors) from the University of Moratuwa, Moratuwa, Sri Lanka, in 1986, the M.Sc. degree in electronics from the University of London, London, U.K., in 1988, and the Ph.D. degree in electrical engineering from the University of Victoria, Victoria, BC, Canada, in 1993.

He was a Postdoctoral Research Fellow with the University of Victoria, Victoria, BC, Canada, from 1993 to 1994 and the University of Bradford, Bradford, U.K., from 1995 to 1996 . He was with Monash University, Melbourne, Australia, from 1997 to 2002. He is currently a Professor with the Department of Electrical and Computer Engineering, University of Alberta, Edmonton, AB, Canada. His research interests include diversity and fading countermeasures, multiple-input-multiple-output systems and space-time coding, and orthogonal frequency division multiplexing.

Prof. Tellambura is an Associate Editor for the IEEE TRANSACTIONS on Communications and the Area Editor for Wireless Communications Systems and Theory in the IEEE TRANSACTIONS ON WIRELESS Communications. He was the Chair of the Communication Theory Symposium in Global Telecommunications Conference in 2005 held in St. Louis, MO. 